\title{
A note on exponents vs root heights for complex simple Lie algebras
}

\author{
Sankaran Viswanath \\ Department of Mathematics \\ University of California \\ Davis, CA 95616, USA \\ svis@math.ucdavis.edu
}

\author{
Submitted: Sep 8, 2006; Accepted: Nov 26, 2006; Published: Dec 7, 2006 \\ Mathematics Subject Classification: 05E15
}

\begin{abstract}
We give an elementary combinatorial proof of a special case of a result due to Bazlov and Ion concerning the Fourier coefficients of the Cherednik kernel. This can be used to give yet another proof of the classical fact that for a complex simple Lie algebra $\mathfrak{g}$, the partition formed by the exponents of $\mathfrak{g}$ is dual to that formed by the numbers of positive roots at each height.
\end{abstract}

Let $\mathfrak{g}$ be a finite dimensional, complex simple Lie algebra of rank $n$ with associated root system $\Delta$, simple roots $\alpha_{i}(i=1 \cdots n)$ and set of positive roots $\Delta^{+}$. Let $Q$ be the root lattice of $\mathfrak{g}$ and $Q^{+}$denote the set comprising $\mathbb{Z}^{\geq 0}$ linear combinations of the $\alpha_{i}$. For each $\alpha \in Q$, let $e^{\alpha}$ denote the corresponding formal exponential; these satisfy the usual rules: $e^{0}=1$ and $e^{\alpha+\beta}=e^{\alpha} e^{\beta}$. We define $\mathcal{A}:=\mathbb{Q}[t]\left[\left[e^{-\alpha_{1}}, \cdots, e^{-\alpha_{n}}\right]\right]$. Thus a typical element of $\mathcal{A}$ is a power series of the form $\sum_{\beta \in Q^{+}} c_{\beta}(t) e^{-\beta}$ where each $c_{\beta}(t) \in \mathbb{Q}[t]$.

Consider the element $\xi \in \mathcal{A}$ defined by:

$$
\begin{aligned}
\xi & :=\prod_{\alpha \in \Delta^{+}} \frac{1-e^{-\alpha}}{1-t e^{-\alpha}} \\
& =\prod_{\alpha \in \Delta^{+}}\left(1+(t-1) e^{-\alpha}+t(t-1) e^{-2 \alpha}+t^{2}(t-1) e^{-3 \alpha}+\cdots\right)
\end{aligned}
$$

Given $\beta=\sum_{i=1}^{n} b_{i} \alpha_{i} \in Q^{+}$, define its height to be

$$
\text { ht } \beta:=\sum_{i=1}^{n} b_{i}
$$

The main objective of this short note is to give an elementary combinatorial proof of the following proposition: 
Proposition 1 For $\beta \in \Delta^{+}$, the coefficient of $e^{-\beta}$ in $\xi$ is $\left(t^{\mathrm{ht}(\beta)}-t^{\mathrm{ht}(\beta)-1}\right)$

This proposition is the $q=0$ case of a more general $(q, t)$ theorem obtained by Bazlov [1] and Ion [2]. They consider

$$
\tilde{K}(q, t)=\prod_{\alpha \in \Delta^{+}} \prod_{i \geq 0} \frac{\left(1-q^{i} e^{-\alpha}\right)\left(1-q^{i+1} e^{\alpha}\right)}{\left(1-t q^{i} e^{-\alpha}\right)\left(1-t q^{i+1} e^{\alpha}\right)}
$$

If $[\tilde{K}(q, t)]$ denotes the constant term (coefficient of $\left.e^{0}\right)$ of $\tilde{K}(q, t)$, one defines $\tilde{C}(q, t):=$ $\tilde{K}(q, t) /[\tilde{K}(q, t)]$ (upto a minor difference in convention, this is called the Cherednik kernel in [2] ). Bazlov and Ion compute the coefficient of $e^{-\beta}$ in $C(q, t)$ for $\beta$ a positive root of $\mathfrak{g}$. Their approaches use techniques from Cherednik's theory of Macdonald polynomials.

When $q=0, \tilde{C}(0, t)$ reduces to $\xi$ introduced above. Though proposition 1 is only a special case, it has a very interesting consequence. Ion showed [2] that it can be used to give a quick and elegant proof of the classical fact that for a finite dimensional simple Lie algebra $\mathfrak{g}$, the partition formed by listing its exponents in descending order is dual to the partition formed by the numbers of positive roots at each height (see below). This fact, first observed empirically by Shapiro and Steinberg was later proved by Kostant [3] using his theory of principal three dimensional subalgebras of $\mathfrak{g}$ and by Macdonald [4] via his factorization of the Poincaré series of the Weyl group of $\mathfrak{g}$.

The motivation for our approach to proposition 1 is to thereby obtain a proof of this classical fact via elementary means (bypassing Macdonald-Cherednik theory).

For completeness sake, we first quickly recall [2] how one can use proposition 1 to deduce the classical fact concerning exponents and heights of roots.

Let $P$ denote the weight lattice of $\mathfrak{g}$ and $W$ its Weyl group. For our definition of exponents, we use the Kostka-Foulkes polynomial $K_{\tilde{\alpha}, 0}(t)$ where $\tilde{\alpha}$ is the highest long root of $\mathfrak{g}$. It is well known that this is given by

$$
K_{\tilde{\alpha}, 0}(t)=\sum_{j=1}^{l} t^{m_{j}}
$$

where $m_{1}, m_{2}, \cdots, m_{l}$ are the exponents of $\mathfrak{g}$. The Kostka-Foulkes polynomials are the elements of the transition matrix between the Schur and the Hall-Littlewood bases of $\mathbb{Q}[t][P]^{W}$. They may be alternatively defined via Lusztig's $t$-analog of weight multiplicity; we have

$$
\begin{aligned}
K_{\tilde{\alpha}, 0}(t) & =\sum_{w \in W}(-1)^{\ell(w)} \mathcal{P}(w(\tilde{\alpha}+\rho)-(0+\rho) ; t) \\
& =\text { coeff. of } e^{0} \text { in } \frac{\sum_{w \in W}(-1)^{\ell(w)} e^{w(\tilde{\alpha}+\rho)-\rho}}{\prod_{\alpha \in \Delta^{+}}\left(1-t e^{-\alpha}\right)}
\end{aligned}
$$


where $\mathcal{P}(; t)$ is the $t$-analog of Kostant's partition function.

The last equation can be rewritten as :

$$
K_{\tilde{\alpha}, 0}(t)=\text { coeff. of } e^{0} \text { in } \frac{\sum_{w \in W}(-1)^{\ell(w)} e^{w(\tilde{\alpha}+\rho)-\rho}}{\prod_{\alpha \in \Delta^{+}}\left(1-e^{-\alpha}\right)} \cdot \xi
$$

where $\xi$ was defined earlier. The expression in (3) (from which we need to extract the coefficient of $e^{0}$ ) is just the product $\chi_{\tilde{\alpha}} \xi$ where $\chi_{\tilde{\alpha}}$ is the formal character of the adjoint representation of $\mathfrak{g}$. This follows from the Weyl character formula and the fact that adjoint representation is irreducible with highest weight $\tilde{\alpha}$. Now,

1. $\chi_{\tilde{\alpha}}=l e^{0}+\sum_{\alpha \in \Delta^{+}}\left(e^{\alpha}+e^{-\alpha}\right)$ and

2. From equation (1), the power series for $\xi$ has constant term 1 and only involves terms of the form $e^{-\gamma}$ for $\gamma \in Q^{+}$.

Thus,

$$
\text { coeff. of } e^{0} \text { in } \chi_{\tilde{\alpha}} \xi=l+\sum_{\alpha \in \Delta^{+}}\left(\text {coeff. of } e^{-\alpha} \text { in } \xi\right)
$$

From proposition 1 , the right hand side equals $l+\sum_{\alpha \in \Delta^{+}}\left(t^{\mathrm{ht}(\alpha)}-t^{\mathrm{ht}(\alpha)-1}\right)$. Letting $a_{i}:=$ $\#\left\{\beta \in \Delta^{+}:\right.$ht $\left.\beta=i\right\}$, this last sum becomes $l+\sum_{i \geq 1} a_{i}\left(t^{i}-t^{i-1}\right)=\left(a_{1}-a_{2}\right) t+\left(a_{2}-a_{3}\right) t^{2}+\cdots\left(\right.$ since $\left.a_{1}=l\right)$. Comparing with equation (2), we get $a_{i}-a_{i+1}$ is the number of times $i$ appears as an exponent of $\mathfrak{g}$. This is exactly the classical result.

Proof of proposition 1: Given $\gamma \in Q^{+}$, let $\operatorname{Par}(\gamma)$ be the set of all partitions of $\gamma$ into a sum of positive roots. Given such a partition $\pi \in \operatorname{Par}(\gamma)$, say

$$
\pi: \quad \gamma=\sum_{\alpha \in \Delta^{+}} c_{\alpha} \alpha \quad\left(c_{\alpha} \in \mathbb{Z}^{\geq 0}\right)
$$

let $n(\pi):=\sum_{\alpha} c_{\alpha}$ be the total number of parts (counting repetitions) and $d(\pi):=\#\{\alpha$ : $\left.c_{\alpha} \neq 0\right\}$ be the number of distinct parts in $\pi$. From equation (1), it is clear that

$$
\text { Coeff. of } e^{-\gamma} \text { in } \xi=\sum_{\pi \in \operatorname{Par}(\gamma)} t^{n(\pi)-d(\pi)}(t-1)^{d(\pi)}
$$




\section{Notation:}

1. Given a subset $A \subset \operatorname{Par}(\gamma)$, let $\operatorname{wt}(A):=\sum_{\pi \in A} t^{n(\pi)-d(\pi)}(t-1)^{d(\pi)}$. Thus the coeff. of $e^{-\gamma}$ in $\xi$ equals wt $(\operatorname{Par}(\gamma))$

2. Given a simple root $\alpha_{i}$, let

$$
\begin{aligned}
& \operatorname{Par}\left(\gamma, \alpha_{i}\right):=\left\{\pi \in \operatorname{Par}(\gamma): \alpha_{i} \text { occurs as one of the parts in } \pi\right\} \\
& \operatorname{Par}\left(\gamma, \widehat{\alpha_{i}}\right):=\left\{\pi \in \operatorname{Par}(\gamma): \alpha_{i} \text { does not occur as a part in } \pi\right\}
\end{aligned}
$$

Let $(\cdot, \cdot)$ denote a nondegenerate, $W$-invariant symmetric bilinear form on the dual of the Cartan subalgebra and let $s_{j} \in W(j=1 \cdots n)$ be the simple reflection correponding to $\alpha_{j}$.

We will prove proposition 1 by induction on ht $\beta$. If ht $\beta=1, \beta$ is a simple root. It is then clear from equation (1) that the coefficient of $e^{-\beta}$ is $t-1=t^{1}-t^{0}$. Now suppose $\beta \in \Delta^{+}$with $h:=$ ht $\beta \geq 2$. Assume the proposition is true for all positive roots of height $<h$. Choose a simple root $\alpha_{i}$ such that $\left(\beta, \alpha_{i}\right)>0\left(\operatorname{such} \alpha_{i}\right.$ exists since $\left.(\beta, \beta)>0\right)$. Now $h \geq 2$ implies that $s_{i} \beta=\beta-2 \frac{\left(\beta, \alpha_{i}\right)}{\left(\alpha_{i}, \alpha_{i}\right)} \alpha_{i}$ is a positive root of height $<h$.

Fact 1: $\operatorname{Par}\left(\beta, \widehat{\alpha}_{i}\right)$ is in bijection with $\operatorname{Par}\left(s_{i} \beta, \widehat{\alpha}_{i}\right)$

Proof: Given a partition $\pi: \beta=\sum_{\alpha \in \Delta^{+}} c_{\alpha} \alpha \in \operatorname{Par}\left(\beta, \widehat{\alpha}_{i}\right)$, we can form a partition of $s_{i} \beta$ as follows:

$$
\tilde{\pi}: s_{i} \beta=\sum_{\alpha \in \Delta^{+}} c_{\alpha}\left(s_{i} \alpha\right)
$$

Since $\alpha_{i}$ is not one of the parts of $\pi$, all the parts of $\tilde{\pi}$ are positive roots, none equal to $\alpha_{i}$. It is clear that $\pi \mapsto \tilde{\pi}$ sets up the required bijection. Further, since $n(\pi)=n(\tilde{\pi})$ and $d(\pi)=d(\tilde{\pi})$, we have

$$
\operatorname{wt}\left(\operatorname{Par}\left(\beta, \widehat{\alpha}_{i}\right)\right)=\operatorname{wt}\left(\operatorname{Par}\left(s_{i} \beta, \widehat{\alpha}_{i}\right)\right)
$$

\section{Fact 2:}

$$
\operatorname{wt}\left(\operatorname{Par}\left(\beta, \alpha_{i}\right)\right)=t \operatorname{wt}\left(\operatorname{Par}\left(\beta-\alpha_{i}, \alpha_{i}\right)\right)+(t-1) \operatorname{wt}\left(\operatorname{Par}\left(\beta-\alpha_{i}, \widehat{\alpha}_{i}\right)\right)
$$

Proof: There is an obvious bijection between the sets $\operatorname{Par}\left(\beta-\alpha_{i}\right)$ and $\operatorname{Par}\left(\beta, \alpha_{i}\right)$ obtained by sending a partition $\pi$ in the first set to the partition $\bar{\pi}$ obtained by adjoining the extra part $\alpha_{i}$ to $\pi$. In order to see how wt $(\pi)$ compares with wt $(\bar{\pi})$, we write $\operatorname{Par}\left(\beta-\alpha_{i}\right)=$ $\operatorname{Par}\left(\beta-\alpha_{i}, \alpha_{i}\right) \cup \operatorname{Par}\left(\beta-\alpha_{i}, \widehat{\alpha}_{i}\right)$. For $\pi \in \operatorname{Par}\left(\beta-\alpha_{i}, \alpha_{i}\right)$, the extra part $\alpha_{i}$ in $\bar{\pi}$ is a repeat part and thus

$$
\mathrm{wt}(\bar{\pi})=t \mathrm{wt}(\pi)
$$

while for $\pi \in \operatorname{Par}\left(\beta-\alpha_{i}, \widehat{\alpha}_{i}\right)$, the extra $\alpha_{i}$ in $\bar{\pi}$ is a new distinct part and thus $\operatorname{wt}(\bar{\pi})=$ $(t-1) \mathrm{wt}(\pi)$. This proves equation $(6)$. 
Let $k:=2 \frac{\left(\beta, \alpha_{i}\right)}{\left(\alpha_{i}, \alpha_{i}\right)}>0$ and consider the $\alpha_{i}$-string through $\beta$ :

$$
\beta, \beta-\alpha_{i}, \cdots, \beta-k \alpha_{i}
$$

Each of these is a positive root. We now rewrite equation (6) as

$$
\operatorname{wt}\left(\operatorname{Par}\left(\beta, \alpha_{i}\right)\right)-\operatorname{wt}\left(\operatorname{Par}\left(\beta-\alpha_{i}, \alpha_{i}\right)\right)=(t-1) \operatorname{wt}\left(\operatorname{Par}\left(\beta-\alpha_{i}\right)\right)
$$

Iterating this equation $k$ times with $\beta-j \alpha_{i}$ in place of $\beta(0 \leq j \leq k-1)$ and summing the resulting equations, we get

$$
\operatorname{wt}\left(\operatorname{Par}\left(\beta, \alpha_{i}\right)\right)-\operatorname{wt}\left(\operatorname{Par}\left(\beta-k \alpha_{i}, \alpha_{i}\right)\right)=(t-1) \sum_{j=1}^{k} \operatorname{wt}\left(\operatorname{Par}\left(\beta-j \alpha_{i}\right)\right)
$$

By induction hypothesis,

$$
\operatorname{wt}\left(\operatorname{Par}\left(\beta-j \alpha_{i}\right)\right)=t^{h-j}-t^{h-j-1}
$$

Further,

$$
\begin{aligned}
\operatorname{wt}\left(\operatorname{Par}\left(\beta-k \alpha_{i}, \alpha_{i}\right)\right) & =\operatorname{wt}\left(\operatorname{Par}\left(\beta-k \alpha_{i}\right)\right)-\operatorname{wt}\left(\operatorname{Par}\left(\beta-k \alpha_{i}, \widehat{\alpha_{i}}\right)\right) \\
& =\left(t^{h-k}-t^{h-k-1}\right)-\operatorname{wt}\left(\operatorname{Par}\left(\beta-k \alpha_{i}, \widehat{\alpha_{i}}\right)\right)
\end{aligned}
$$

Since $\beta-k \alpha_{i}=s_{i} \beta$, we can use equation (5). This gives

$$
\begin{aligned}
\mathrm{wt}\left(\operatorname{Par}\left(\beta, \alpha_{i}\right)\right)+\mathrm{wt}\left(\operatorname{Par}\left(\beta, \widehat{\alpha_{i}}\right)\right) & =(t-1) \sum_{j=1}^{k}\left(t^{h-j}-t^{h-j-1}\right)+\left(t^{h-k}-t^{h-k-1}\right) \\
& =t^{h}-t^{h-1}
\end{aligned}
$$

Since the left hand side equals wt $(\operatorname{Par}(\beta))$, proposition 1 is proved.

Acknowledgements : The author would like to thank John Stembridge for bringing references [1] and [2] to his attention and for his comments on an earlier draft of this note.

\section{References}

[1] Yuri Bazlov. Graded multiplicities in the exterior algebra. Adv. Math., 158(2):129-153, 2001.

[2] Bogdan Ion. The Cherednik kernel and generalized exponents. Int. Math. Res. Not., (36):1869-1895, 2004.

[3] Bertram Kostant. The principal three-dimensional subgroup and the Betti numbers of a complex simple Lie group. Amer. J. Math., 81:973-1032, 1959.

[4] I. G. Macdonald. The Poincaré series of a Coxeter group. Math. Ann., 199:161-174, 1972. 